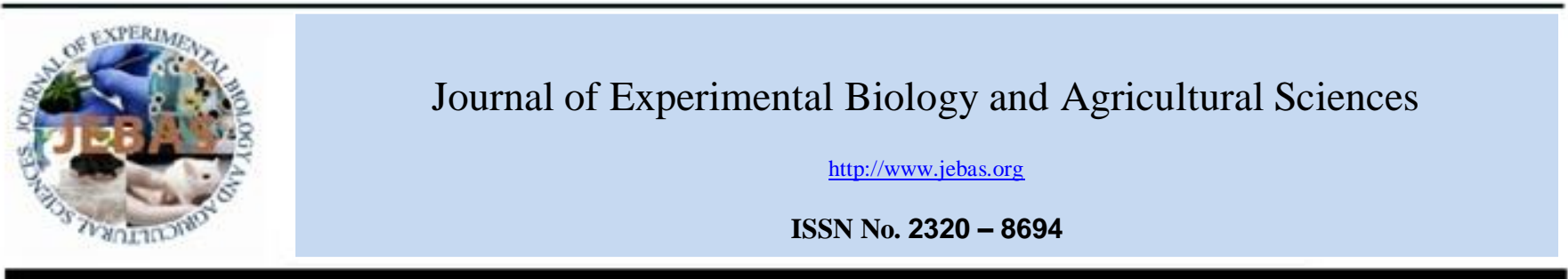

\title{
COMPARATIVE ANALYSIS OF GENE EXPRESSION PROFILES AMONG CONTRASTING MULBERRY VARIETIES UNDER COLD STRESS CONDITION
}

\author{
Pawan Shukla ${ }^{1 \mathrm{a} *}$, Ramesha A. Reddy ${ }^{2 \mathrm{a}}$, Kangayam M. Ponnuvel ${ }^{2}$, Gulab Khan Rohela ${ }^{1}$, \\ Aftab Ahmad Shabnam ${ }^{1}$, S. S. Chauhan ${ }^{1}$, Mrinal Kanti Ghosh ${ }^{1}$, Rakesh Kumar Mishra ${ }^{2}$ \\ ${ }^{1}$ Central Sericultural Research and Training Institute, Central Silk Board, NH-1A, Gallandar, Pampore - 192 121, J \& K, India \\ ${ }^{2}$ Seri-Biotech Research Laboratory (SBRL), Kodathi, Carmelram Post, Bangalore - 560035 \\ a authors contributed equally to this work.
}

Received - October 20, 2018; Revision - December 02, 2018; Accepted - December 12, 2018

Available Online - December 15, 2018

DOI: http://dx.doi.org/10.18006/2018.6(6).973.982

KEYWORDS

Mulberry

Cold stress

WRKY46

Cysteine proteinase inhibitor

Spermidine synthase

COR413

TIFY10

ERD10

\begin{abstract}
Low temperature/ cold stress is a major abiotic stress affecting mulberry plant growth and productivity in temperate climatic conditions. Mulberry leaf is a sole food source for the economically important insect, Bombyx mori. Cold stress severely damages buds and young leaves and affecting growth and leaf productivity. Therefore, it is required to identify genes which are responsive and impart cold tolerance and utilize them to develop improved mulberry varieties, which can withstand cold stress without compromising its leaf production. In the present study, candidate cold tolerant gene sequences of WRKY46, Spermidine synthase, early response to dehydration10 (ERD10), TIFY10, Cysteine proteinase Inhibitor (CPI), Cold regulated 413 plasma membrane protein 2 (COR413) were retrieved from the mulberry database through NCBI and bioinformatics tools. Comparative gene expression analysis was performed in temperate mulberry varieties, Gosherami, Leh 2 (saspole) and Gurez using tropical variety, G4 as a reference in cold stress condition. Results depicted that WRKY46, Spermidine synthase, ERD10, TIFY10, CPI, COR413 genes showed significant up-regulation under cold stress condition. Gurez genotype displayed significant up-regulation of these selected cold tolerant genes under both laboratory and field conditions compared to other mulberry genotypes.
\end{abstract}

* Corresponding author

E-mail: shklpwn@gmail.com (Pawan Shukla)

Peer review under responsibility of Journal of Experimental Biology and Agricultural Sciences.

Production and Hosting by Horizon Publisher India [HPI] (http://www.horizonpublisherindia.in/).

All rights reserved.
All the article published by Journal of Experimental Biology and Agricultural Sciences is licensed under a Creative Commons Attribution-NonCommercial 4.0 International License Based on a work at www.jebas.org.

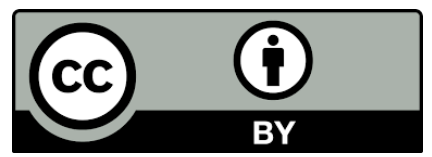




\section{Introduction}

Plants grown under temperate climatic condition faced freezing or extreme low temperature condition. In general, plants from temperate climate are found to be chilling tolerant with variable degree, and can increase their freezing tolerance by being exposed to chilling, non-freezing temperatures. These temperate plants have evolved a mechanism via cold acclimation to enhance tolerance to low temperatures (Thomashow, 1999). In addition, the same mechanism used by perennial woody plant species of temperate regions to tolerate freezing (Levitt, 1980; Sakai \& Larcher, 1987). However, the high freezing tolerance exhibited by perennial woody plants is regulated in response to cues such as the short day and decreased air temperatures during autumn (Sakai \& Larcher, 1987). In many herbaceous and perennial woody plants, including mulberry, freezing tolerance is dependent on occurrence of numerous biochemical and physiological changes (Sakai \&Yoshida, 1968; Yoshida, 1984; Fujikawa, 1994). These changes brought at cellular level through compositional changes in the plasma membranes (Steponkus, 1984; Yoshida, 1984; Uemura et al., 1995), enhanced accumulation of compatible osmolytes, such as soluble sugars, prolines, and betaines (Hare et al., 1998), induction of gene expression such as heat shock proteins (Neven et al., 1992; Ukaji et al., 1999), cold-regulated (COR) proteins (Guy et al., 1985; Thomashow, 1999), increased extracellular accumulation of antifreeze proteins (Griffith \& Antikainen, 1996), and changes in the property of cell walls (Rajashekar \& Lafta, 1996; Fujikawa and Kuroda, 2000) were found to be associated with freezing tolerance (Guy, 1990; Fujikawa et al., 1999; Thomashow, 1999).

Genes such as WRKY46, Spermidine synthase, early response to dehydration10 (ERD10), TIFY10, Cysteine proteinase Inhibitor (CPI), cold regulated 413 plasma membrane protein 2 (COR413) have been shown to be involved in cold stress acclimation in different model and crop plants (Martin-Tanguy, 2001; Breton et al., 2003; Puhakainen et al., 2004; Wang et al., 2012; Huang et al., 2016). WRKYs are transcription factors which regulate numerous downstream genes involved in stress adaptation by binding to $\mathrm{W}$ box sequences present in the promoter region. WRKY genes were reported to be involved in different stresses and shown to be upregulated during cold stress in mulberry (Wang et al., 2012; Ding et al., 2014; Baranwal et al., 2016). Dehydrin belongs to the group 2 LEA (Late Embryogenesis Abundant II) proteins. These proteins accumulate in late embryogenesis or can be induced in response to dehydration, salt, cold and freezing stress (Wisniewski et al., 1999; Puhakainen et al., 2004; Rorat et al., 2006; Hanin et al., 2011). Spermidine synthase is a key enzyme in polyamine spermidine biosynthesis (Kasukabe et al., 2004). Polyamine spermidines are low molecular weight aliphatic amines and play an important role in growth, development reproduction and stress adaptation in plants (Kakkar et al., 2000; Martin-Tanguy, 2001).

The TIFY are novel plant specific genes are characterized by a conserved TIFY motif (TIFF/YXG) (Zhu et al., 2014). TIFY genes were reported to involve in jasmonate signaling, plant growth, development, pathogen response and in response to abiotic stress (Zhu et al., 2011; Hakata et al., 2012; Zhu et al., 2012). Upregulation of TIFY genes under cold stress was reported in moso bamboo (Phyllostachys edulis) (Huang et al., 2016) and rice (Ye et al., 2009). Cysteine proteinase inhibitors, small peptides of 12-16 $\mathrm{kDa}$, are proteinaceous inhibitor of papain (Benchabane et al., 2010). Reports suggests that cysteine proteinase inhibitor play an important role in plants exposed to different stresses including cold stress (Zhang et al., 2008; Benchabane et al., 2010; Kunert et al., 2015; Subburaj et al., 2017).

Cold regulated 413 plasma membrane protein 2 belongs to COR413 family and having five putative transmembrane domain (Breton et al., 2003). Cold regulated 413 plasma membrane protein 2 (COR413-PM2) was first reported in wheat and Arabidopsis and accumulation of its transcript correlated with freezing tolerance in these plants (Breton et al., 2003).

Sericulture is practiced in temperate climatic conditions where, mulberry, the sole food source for the silkworm, Bombyx mori encounters cold stress (Rohela et al., 2016; Rohela et al., 2018a; Rohela et al., 2018b; Shabnam et al., 2018). Due to cold/frost, upper branch of the mulberry plant get damage which in turn affect the leaf yield for the spring rearing of silkworm (Shukla et al. 2016a). To the extent of $30 \%$ frost damage of mulberry plant was reported during temperate climate of Kashmir valley, India (Ahanger et al., 2013). This is due to existing genotypes used in sericulture industry are not frost tolerant. However few genotypes from Leh, Ladakh and Gurez regions, India displayed high degree of cold adaptation, but they are not commercially exploited. The molecular mechanism of strong adaptability and tolerance to cold stress has not been studied for these genotypes, which limits its exploitation. Therefore, it is required to understand the genes involved in cold tolerance mechanism in these mulberry genotypes to utilize in mulberry breeding.

In present study, a comparative gene expression study of WRKY46, Spermidine synthase, Early response to dehydration 10 (ERD10), TIFY10, Cysteine proteinase Inhibitor (CPI), Cold regulated 413 plasma membrane protein 2 (COR413) was performed in temperate mulberry, Gosherami, Leh 2 (Saspole) and Gurez along with tropical mulberry germplasm G4 and V1 in cold stress condition. Present study was carried out with an aim to observe the expression pattern of these genes under cold stress condition for their utilization in mulberry improvement. 


\section{Materials and Methods}

2.1 Retrieval of gene sequences, Multiple Sequence Alignment, Phylogenetic analysis and Analysis of gene structure

Putative cold tolerant gene sequences were retrieved from the Mulberry genome database MorusDB and comparative sequences analysis using NCBI-BLAST. Similar, cold tolerant gene sequences were also retrieved from other plant species. These sequences were arranged in fasta format and multiple sequence alignment was performed using MUSCLE (https://www.ebi.ac.uk/Tools/msa/muscle/). Based on the alignment, the phylogenetic tree was constructed from the amino acid sequences using the neighbor-joining (NJ) method in MEGA 7 with 1000 bootstrap replicates. The exon-intron structures of the gene sequences were determined using Gene Structure Display Server (GSDS:http://gsds.cbi.pku.edu.ch) by aligning cDNA to their corresponding genomic DNA sequences.

\subsection{Plant materials and cold stress treatments}

Mulberry (Morus alba) variety G4 grown in nursery and transplanted to pots. Similarly, Gosherami, Leh 2 (Saspole) and Gurez variety were grown, buds from these varieties were grafted on to G4. These bud grafted cuttings were grown under well watering condition in plastic pots for three months. For cold treatments, plants were transferred to incubator at $4^{\circ} \mathrm{C}$ and samples were collected at $6 \mathrm{hrs}, 24 \mathrm{hrs}$ and $48 \mathrm{hrs}$.

\subsection{RNA extraction and cDNA synthesis}

Total RNA extraction was performed as per the manufacturer's instructions using Nuceospin plant RNA extraction kit (TaKaRa, Japan). Quantification of RNA samples were determined using the NanoDrop 2000c spectrophotometer (Thermo Fisher Scientific, USA) and the quality of RNA samples was analyzed on ethidium bromide stained-agarose gels. First-strand cDNA synthesis was performed according to manufacturer's instructions through the PrimeScript 1st strand cDNA synthesis kit (TaKaRa, Japan). Further, 10 fold dilution of cDNA was carried out with nuclease free sterile water for qRT-PCR (Shukla et al., 2016a; Ahmed et al., 2018).

\subsection{Primer design}

Primers for expression study were performed using Primer-Blast software (www.ncbi.nlm.nih.gov/tools/primer-blast) with product size of 100-180 bp and primer length 20-25 bp. The details of the primers used in this study are given in Table 1.

\subsection{Quantitative Real Time PCR}

Quantitative real time PCR (qPCR) was performed using SYBR ${ }^{\circledR}$ Premix Ex Taq тм II (Tli RNaseH Plus) (TaKaRa, Japan)on Stratagene Mx3005P (Agilent Technologies). A reaction mixture of $10 \mu \mathrm{l}$ was prepared having cDNA template $(1 \mu \mathrm{l})$, primer (0.2mM forward \& reverse each), and SYBR Premix Ex Taq II (1X). The PCR was set in a 96 -well reaction plate at $94^{\circ} \mathrm{C}$ for 30 s, followed by 40 cycles of $94^{\circ} \mathrm{C}$ for $10 \mathrm{~s}, 55^{\circ} \mathrm{C}$ for $30 \mathrm{~s}$, and $72^{\circ} \mathrm{C}$ for $30 \mathrm{~s}$. After 40 cycles, the melting curve was determined to verify primer specificity by heating from $65^{\circ} \mathrm{C}$ to $95^{\circ} \mathrm{C}$. Each qPCR reaction was performed in two technical replicates and three biological replicates (Ahmed et al. 2018).

Table 1 Details of primers sequences used for the study

\begin{tabular}{|ccc|}
\hline S. No. & Name & Sequence $\left(5^{\prime}-3^{\prime}\right)$ \\
\hline 1. & WRKY46 qRT Forward & GAGGTCACTTACAGAGGCCG \\
\hline 2. & WRKY46 qRT Reverse & GACCTGTTGCTTGTGGTTGC \\
\hline 3. & ERD10 qRT Forward & CTATCGCCGGTGACTTCGAG \\
\hline 4. & ERD10 qRT Reverse & AACCGGGCTTTTTCTCCTCC \\
\hline 5. & TIFY10 qRT Forward & CGGTTCTTGGAGAAGAGAAAG \\
\hline 6. & TIFY10 qRTReverse & TCATTGGGTTTGTTGAGCAGC \\
\hline 7. & Spermidine qRTForward & AAGTTGCTCGGCATACCTCC \\
\hline 8. & Spermidine qRT Reverse & GGGACAGCCTTCAAAAAGGC \\
\hline 9. & COR413 qRT Forward & CAAGCTGAAAACCAGGGCAC \\
\hline 10. & COR413qRT Reverse & CCCTCCCCATTTCAGCTCTC \\
\hline 11. & CPI qRT Forward & GAAACTCGGCGGAATCCAAG \\
\hline 12. & CPI qRT Reverse & AGACCGTACCGGCAACAAC \\
\hline
\end{tabular}

Journal of Experimental Biology and Agricultural Sciences http://www.jebas.org 


\section{Results}

3.1 Identification of candidate cold tolerant gene sequences from mulberry

WRKY46, Spermidine synthase, CPI, ERD10, COR413, TIFY10 gene sequences were retrieved from MorusDB using NCBIBLAST. M. notabilis used as a reference for identification of candidate cold tolerant genes from mulberry as whole genome sequence and annotation is publicly available. Nucleotide and amino acid sequence of M. notabilis WRKY46 was 1095 bp and 364 aa respectively (accession no. XM_010097487.1). Similar gene sequence was also identified from $M$. alba (accession no. KP765436.1), which exhibited $96.99 \%$ nucleotide and $94.51 \%$ amino acid identity with $M$. notabilis sequence. Spermidine synthase gene from $M$. notabilis codes for 1005 bp nucleotide sequence and 334 aa (accession no. XM_010102183.1). Similar, but partial sequence of spermidine synthase was also identified from M. nigra (accession no. KP980553.1). Cysteine proteinase inhibitor of $306 \mathrm{bp}$ and 101 aa was also fished out from $M$. notabilis (accession no. XM_010108020.1) and $M$. alba (accession no. KM091230.1) that exhibited $99.35 \%$ and $99.01 \%$ nucleotide and amino acid identity respectively. ERD10 gene was identified as 759 bp and 252 aa. M.notabilis COR413 gene sequence was identified having $606 \mathrm{bp}$ nucleotide and 201 aa sequence. TIFY10A contained 822 bp and 273 aa (accession no. XM_010100794.1) of nucleotide and amino acid sequence respectively. EST sequence showing identity with TIFY10A (ES448372.1), WRKY46 (ES448888.1), spermidine synthase (JK705754.1), cysteine proteinase inhibitor (ES449068.1) were also retrieved from the EST database of mature leaf of $M$. indica.
3.2 Multiple sequence alignment, Phylogenetic analysis, and gene structure of cold tolerant genes

Multiple sequence alignment of WRKY46 of different plant species revealed that $M$. notabilis WRKY46 shows $55.21 \%, 41.52 \%, 39.52 \%$, $30.59 \%$, 28.57\%, 27.85\% similarity with Populus trichocarpa, Brassica rapa, Arabidopsis thaliana, Gossypium hirsutum, Zea mays, Triticum aestivum respectively and having conserved WRKY specific domain. Phylogenetic analysis also showed that $M$. notabilis displayed highest similarity with M. alba and Populus trichocarpa. Alignment of M. notabilis CPI revealed the presence of inhibitory domain and showed overall similarity of $99.01 \%, 70.10 \%, 69.70 \%, 66.00 \%$, $63.00 \%$ with M. alba, Coffea canephora, Boehmeria nivea, Cajanaus cajan, Hevea brasiliensis, respectively. Similar results were observed with phylogenetic analysis. Sequence alignment and phylogenetic analysis of M. notabilis ERD10 showed highest similarity with Hevea brasiliensis and presence S-segment (SSSSSS) and K-segment (EKIKEKLPG) specific to ERD10. Sequence alignment of TIFY 10A showed the presence of TIFY motif (TIFF/YXG) and Jas domain. Sequence alignment and phylogenetic analysis of $M$. notabilis TIFY10A with different plant species showed highest similarity with Malus domestica. Multiple sequence alignment and phylogenetic analysis of Morus spermidine synthase gene sequences with different species shows its close similarity with Populus trichocarpa (86.03\%) and Malus domestica (84.76\%). COR413 gene sequence alignment with other species shows close identity with Hevea brasiliensis (88.56\%) and Malus domestica (84.08\%). Phylogenetic analysis of COR413 also revealed the same result. The gene structure features like exon-intron distribution and conserved motif of M. notabilis WRKY46, Spermidine synthase, Cysteine proteinase inhibitor, ERD10, COR413, TIFY10 gene sequences were analyzed. ERD10 and cysteine proteinase inhibitor contain 02 exons, WRKY46 contain 3 exons, COR413 contain 04 exons, TIFY10A with 05 exons, spermidine synthase contain 09 exons (Figure 1).

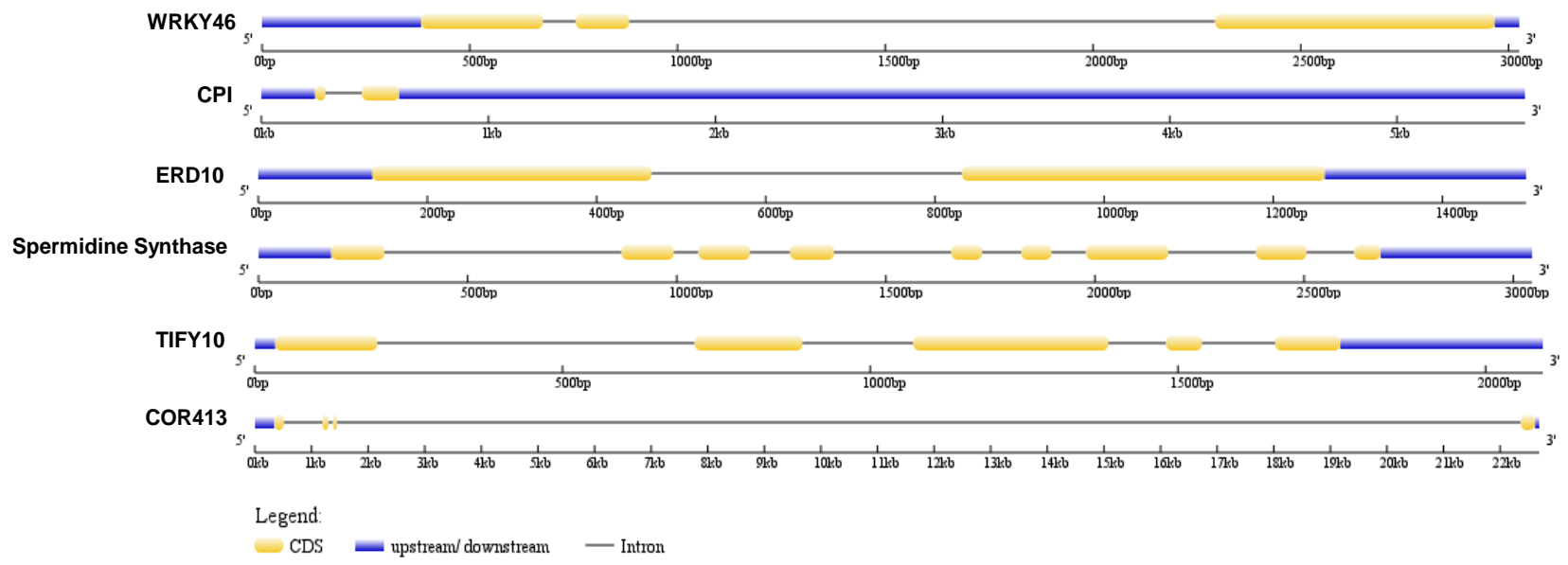

Figure 1 Gene Structure of WRKY46, CPI, ERD10, Spermidine synthase, TIFY10, COR413.

Journal of Experimental Biology and Agricultural Sciences

http://www.jebas.org 
3.3 Comparative expression profile of candidate genes in cold stress condition:

Quantitative PCR was performed for the in silico identified cold tolerant genes and relative expression analysis was carried out in tropical (G4 genotype) and temperate mulberry genotypes (Gosherami, Leh 2 (Saspol) and Gurez) upon exposure to cold stress. Comparative expression profile performed by two methods, where control samples without stress from the respective genotypes used as a reference and in other method, expression levels of tropical genotype, G4 as a reference sample. It was observed that in silico identified putative cold tolerant genes like WRKY46, Spermidine synthase, ERD1, TIFY10,CPI, COR413 displayed upregulation during cold stress condition. WRKY46 gene displayed nearly 31 fold upregulation in Gosherami, leh 2 and gurez genotype while 6 fold in G4 genotype compared to untreated samples (Figure 2 A). But, WRKY46 gene showed 5.8 fold upregulation in gurez genotype, 2.4 fold upregulation in Gosherami and 1.8 fold upregulation in Leh 2 genotype when G4 expression used as a control (Figure 2G).Under cold stress condition, Spermidine synthase was found to be upregulated 16.7 fold in Gurez, 15.7 fold in Gosherami, 3.85 fold in G4 and 2.74 fold in Leh 2 compared to untreated sample as a control in contrast to G4 used as a control (Figure 2B). Spermidine synthase expression was downregulated in Gosherami and Leh 2 while 5.7 fold upregulated in Gurez (Figure 2H). ERD10 expression was 129 fold in Gosherami, 24 fold in Gurez, 2.5 fold in G4 and down regulated in Leh2 genotype compare to untreated sample as a control (Figure 2C). The expression of ERD10 induced to 351 fold in Gosherami, 7.4 fold in Gurez and 5.6 fold in Leh 2 when G4 used as a control (Figure 2I). TIFY10 gene expression was highest in Gurez 144.76 fold followed by Gosherami 102 fold and in G4 10.87 fold and Leh 2, with 5.7 fold when untreated sample used as control (Figure 2D). When G4 used as a control, Gurez displayed highest expression of 6.7 fold while Gosherami and Leh2 showed 2.9 and 2.0 fold respectively (Figure 2J). CPI expression compared to untreated control was 9.66 fold in Gosherami, 8.77 fold in Gurez, 4.71 fold in leh 2 and 1.86 fold in G4 genotype (Figure 2E). When data was compared with G4 as a control, Gurez showed highest expression of 9.6 fold, followed by Leh 2 with 4 fold and Gosherami with 2.8 fold (Figure 2K). COR413 expression was observed highest 12 fold in Gurez, 3 fold in Gosherami and 2 fold in Leh 2 and G4 under cold stress condition when untreated sample used as a control (Figure 2F) but when G4 used as a control only gurez displayed 9.5 fold expression of COR413 while downregulation of COR413 expression was observed in Gosherami and Leh 2 (Figure 2L).
3.4 Comparative expression profile of candidate cold tolerant genes under field conditions

Laboratory and controlled conditions experiment results suggest that Gurez genotype exhibited highest up-regulation of most of the tested cold tolerant genes compared to Gosherami and Leh2 genotype. To further validate the expression profile in Gurez, RNA was isolated from the sample collected from the tropical mulberry variety, V1, and temperate varieties, Narula and Gurez under field cold stress condition $\left(6^{\circ} \mathrm{C} \pm 2\right)$ from the mulberry germplasm bank at CSR\&TI, Pampore, India. The relative expression of selected genes was analyzed in these samples in which V1 was used as reference for normalizing the relative expression of genes. Upregulation of WRKY46, Spermidinesynthase, CPI, ERD1, COR413, TIFY10 was observed in Gurez genotype compare to Narula genotype. Expression of WRKY46 was 22 fold in gurez while 3 fold in Narula, Spermidine synthase expression was 7 fold in gurez while 4 fold in narula, CPI expression was 15.5 fold in gurez while 4 fold in narula, ERD10 expression was 4.6 fold in gurez while 2 fold in narula, COR413 expression 9 fold in gurez while 7 fold in narula, TIFY10 expression 13.5 fold in gurez while 5.7 fold in narula (Figure 3).

\section{Discussion and conclusions:}

Freezing or extremely low temperature constitutes a key factor in temperate region by influencing plant growth, development and productivity. Plants in temperate region of Kashmir affected by cold stress especially mulberry plants, which is important for sericulture Industry (Shukla et al., 2016b). Kashmir valley is famous for its bivoltine temperate sericulture. However, being a temperate region, it is important to mention that the mulberry genotypes used for sericulture were affected by frost/cold damage. However, there are genotypes identified from Leh, ladakh and gurez region that displayed high degree of tolerance to cold. Understanding the mechanism of tolerance and genes involved in cold tolerance can be useful for development of cold tolerant varieties. Mulberry genome (M. notabilis) was sequenced in 2013 and in addition, large amount of transcriptomic data of $M$. notabilis (a haploid genome $\mathrm{n}=7$ ) was generated (He et al., 2013). These data are increasingly used for comparative genomics, identification of many useful genes and analysis of non-coding sequences (He et al., 2013; Ma et al., 2014; Wang et al., 2014; Wei et al., 2014; Song et al., 2016;). Recently, Transcriptomic data from M. laevigata and M. serrata was generated (Saeed et al., 2016) and utilized for identification and genome wide analysis of WRKY and NAC transcription factor (Baranwal \& Khurana, 2016; Baranwal et al., 2016). 
A.

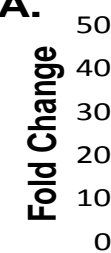

B.

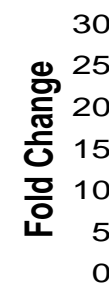

C.

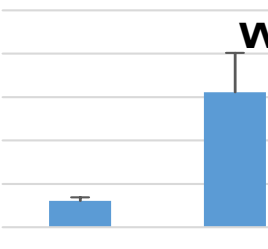

WRKY 46

GC

OC

LC

ZC

\section{Spermidine synthase}

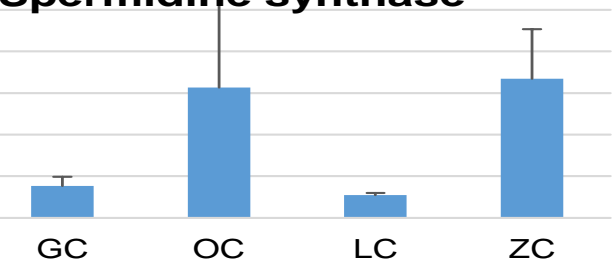

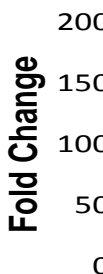

D.

GC
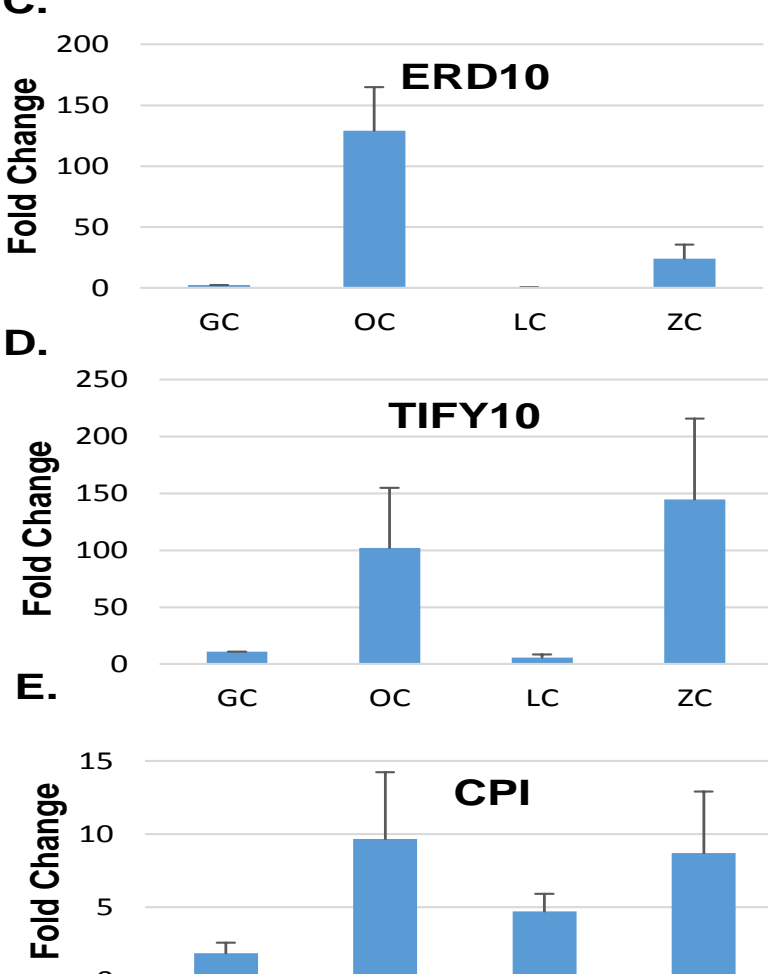

F.
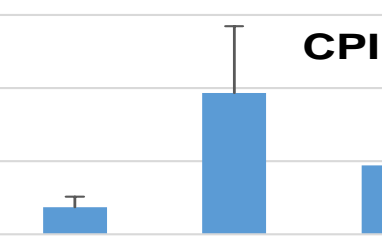

GC

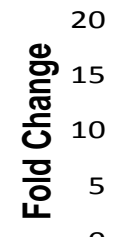

OC

ZC

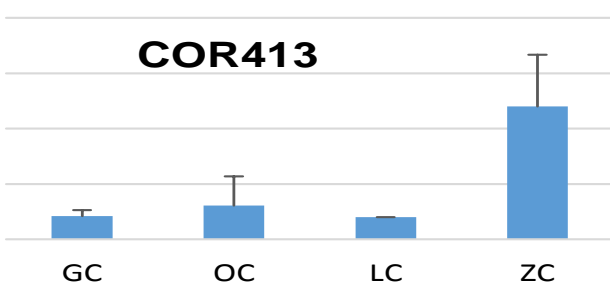

G.

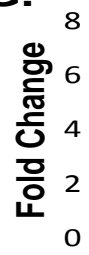

H.

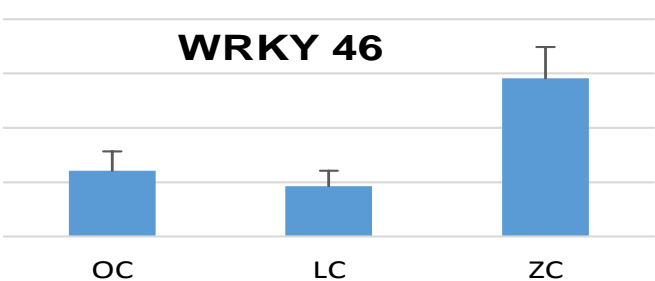

H.

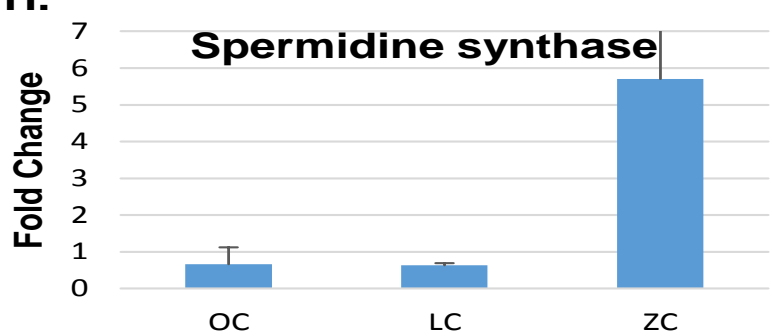

I.

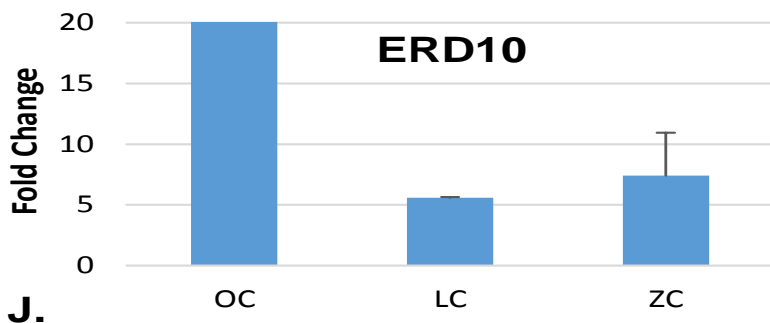

10

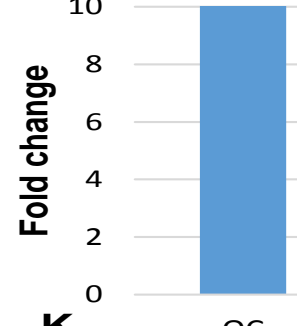

TIFY10

K.

OC

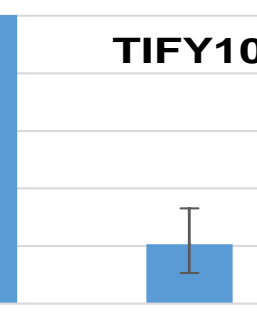

LC

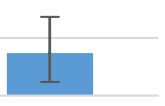

ZC

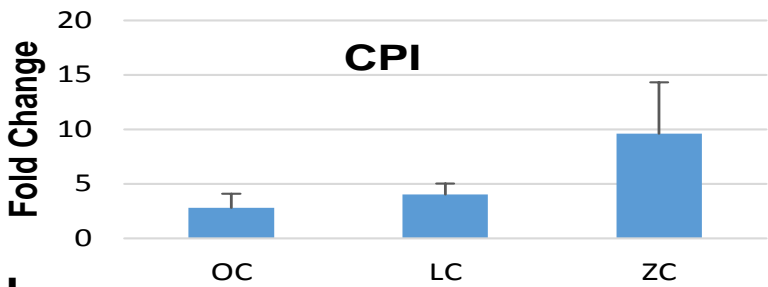

L.
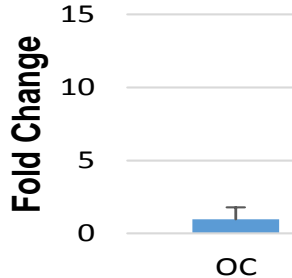

C0413

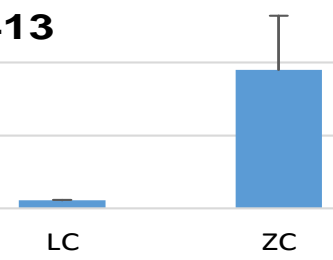

Figure 2: Relative expression of WRKY46, Spermidine synthase, ERD10, TIFY10, CPI, COR413 under cold stress under laboratory conditions. The relative expression of genes in cold stress as compared un-treated samples as control (A, B, C, D, E \& F). The relative gene expression of genes compared to cold stress treated G4 samples as control (G, H, I, J, K \& L). GC: G4; OC: Gosherami; LC: Leh 2; ZC: Gurez. 

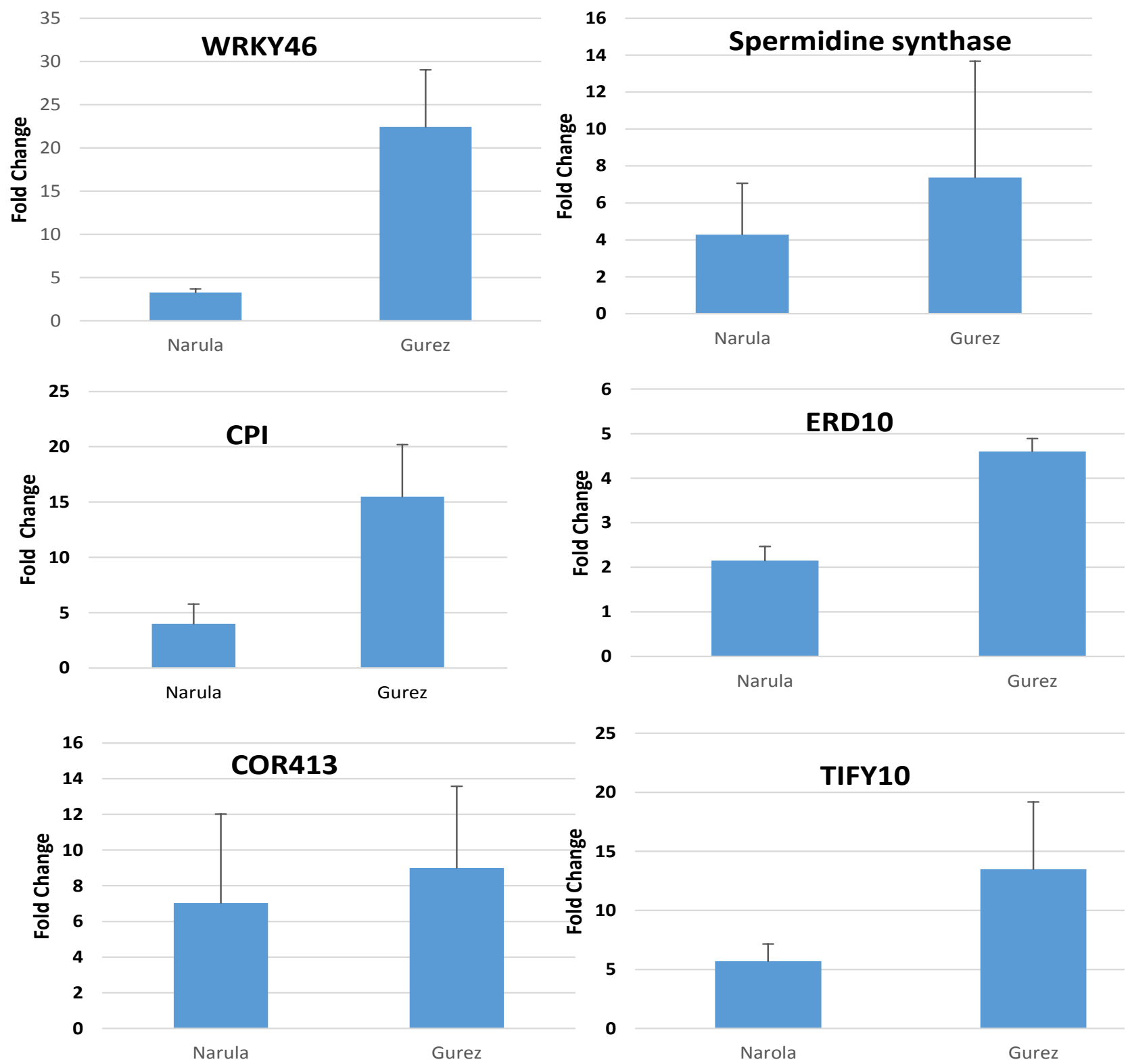

Figure 3 Relative expression of WRKY46, Spermidine synthase, ERD10, TIFY10, CPI, COR413 under cold stress from field conditions. Leaf samples of narula and gurez were collected from Germplasm Bank of CSR\&TI, Pampore under low tempreture condition (Temperature: $6^{\circ} \mathrm{C} \pm 2$ ).

Results of present study indicated the up-regulation of WRKY46, ERD10, spermidine synthase, TIFY10, Cysteine proteinase inhibitor, and COR413 when exposed to cold stress condition both in laboratory and field conditions. Many reports also showed the up-regulation of these genes under cold stress condition in different crop plants. For instance, Brassica compestris WRKY46 (bcWRKY46) was previously reported to enhance cold tolerance in transgenic tobacco. Baranwal et al. (2016) reported the differential regulation of mulberry WRKY genes under different abiotic stress conditions including cold stress. Dehydrins like DHN, ERD10, LEA etc. were reported to be enhance freezing tolerance in Arabidopsis and also involve in different abiotic stress tolerance (Puhakainen et al., 2004; Rorat et al., 2006; Hanin et al., 2011). Spermidine synthase was found to involve in plant growth and development (Kakkar et al., 2000; Martin-Tanguy, 2001) and also provide chilling tolerance in cucumber cultivar (Shen et al., 2000). 
Previous study reported the involvement of TIFY 10 in alkaline stress (Zhu et al., 2014), while in present study up-regulation of TIFY10 expression during cold stress in different mulberry genotypes was reported. This indicated that this gene might also involve in cold stress tolerance. Cysteine proteinase inhibitor (CPI) genes are expressed in plants exposed to high salt, drought, cold and heat stress (Zhang et al., 2008; Je et al., 2014; Kunert et al., 2015). In Arabidopsis, expression of cysteine proteinase inhibitor conferred the cold tolerance in transgenic plants (Zhang et al., 2008). Cold regulated 413 plasma membrane protein 2 transcript accumulation was correlated with freezing tolerance in Arabidopsis and wheat (Breton et al., 2003). In Brachypodium, accumulation of BradiCOR413 transcripts was reported after cold treatment (Colton-Gagnon et al., 2013). Over expression of tomato SICOR413IM1 improved chilling tolerance in transgenic plants (Ma et al., 2018).

Comparison of candidate cold tolerant gene expression profile between genotypes found that temperate genotypes showed higher gene expression levels compared to tropical genotypes. Among the temperate genotypes, gurez genotype displayed the highest level of cold tolerant gene expression. These results suggest that there is a genetic and molecular difference between genotypes for acclimation to cold stress resulting from differential expression and regulation of cold tolerant genes.

In conclusion, the analyzed candidate genes are induced in response to cold stress and among the genotypes Gurez displayed highest upregulation under both laboratory and field conditions suggests these genes might involve in cold tolerance in this genotype. Further, these genes from Gurez genotype are good candidate for development of cold tolerant mulberry varieties.

\section{Acknowledgements}

The authors are thankful to Central Silk Board and CSR \& TI, Pampore for providing a research grant in the form of Project (Project code: PIB-3579) and Seri-Biotech Research laboratory (SBRL), Kodathi, Bangalore for providing facilities to carry out the study.

Conflict of Interest: There is no conflict of Interest.

\section{References}

Ahanger M, Ramegowda G, Illahi I, Rizvi G, Dhar A, Sahaf K (2013) Reaction of Mulberry germplasm to frost damage in Kashmir. Research Journal of Agricultural Sciences 4:180-183.

Ahmed I, Yadav D, Shukla P, Kirti PB (2018) Heterologous expression of Brassica juncea annexin, AnnBj2 confers salt tolerance and $\mathrm{ABA}$ insensitivity in transgenic tobacco seedlings. Functional \& Integrative Genomics, 1-11.
Baranwal VK, Khurana P (2016) Genome-wide analysis, expression dynamics and varietal comparison of NAC gene family at various developmental stages in Morus notabilis. Molecular Genetics Genomics 291:1305-17.

Baranwal VK, Negi N, Khurana P (2016) Genome-wide Identification and Structural, Functional and Evolutionary Analysis of WRKY Components of Mulberry. Scientific Report 6:30794.

Benchabane M, Schluter U, Vorster J, Goulet MC, Michaud D (2010) Plant cystatins. Biochimie 92:1657-66.

Breton G, Danyluk J, Charron JBF, Sarhan F (2003) Expression profiling and bioinformatic analyses of a novel stress-regulated multispanning transmembrane protein family from cereals and Arabidopsis. Plant Physiology 132:64-74.

Colton-Gagnon K, Ali-Benali MA, Mayer BF, Dionne R, Bertrand A, Do Carmo S, Charron JB (2013) Comparative analysis of the cold acclimation and freezing tolerance capacities of seven diploid Brachypodium distachyon accessions. Annals of Botany 113:681-693.

Ding ZJ, Yan JY, Xu XY, Yu DQ, Li GX, Zhang SQ, Zheng SJ (2014) Transcription factor WRKY 46 regulates osmotic stress responses and stomatal movement independently in A rabidopsis. The Plant Journal 79:13-27.

Fujikawa S (1994) Seasonal ultrastructural alterations in the plasma membrane produced by slow freezing in cortical tissues of mulberry (Morus bombyciz Koidz. cv. Goroji). Trees 8:288-296.

Fujikawa S, Jitsuyama Y, Kuroda K (1999) Determination of the role of cold acclimation-induced diverse changes in plant cells from the viewpoint of avoidance of freezing injury. Journal of Plant Research 112:237-244.

Fujikawa S, Kuroda K (2000) Cryo-scanning electron microscopic study on freezing behavior of xylem ray parenchyma cells in hardwood species. Micron 31:669-686.

Griffith M, Antikainen M (1996) Extracellular ice formation in freezing-tolerant plants. Advances in Low-Temperature Biology 3:107-139.

Guy C, Niemi KJ, Brambl R (1985) Altered gene expression during cold acclimation of spinach. Proceedings of the National Academy of Sciences 82:3673-3677.

Guy CL (1990) Cold accelimation and freezing stress tolerance: role of protein metabolism. Annual review of plant biology 41:187-223. 
Hakata M, Kuroda M, Ohsumi A, Hirose T, Nakamura H, Muramatsu M, Ichikawa H, Yamakawa H (2012) Overexpression of a rice TIFY gene increases grain size through enhanced accumulation of carbohydrates in the stem. Bioscience, Biotechnology and Biochemistry 76:2129-2134.

Hanin M, Brini F, Ebel C, Toda Y, Takeda S, Masmoudi K (2011) Plant dehydrins and stress tolerance: versatile proteins for complex mechanisms. Plant Signal \& Behavior 6:1503-1509.

Hare PD, Cress WA, Van Staden J (1998) Dissecting the roles of osmolyte accumulation during stress. Plant, Cell \& Environment 21:535-553.

He N, Zhang C, Qi X, Zhao S, Tao Y, Yang G, Lee TH, Wang X, Cai Q, Li D, Lu M, Liao S, Luo G, He R, Tan X, Xu Y, Li T, Zhao A, Jia L, Fu Q, Zeng Q, Gao C, Ma B, Liang J, Shang J, Song P, Wu H, Fan L, Wang Q, Shuai Q, Zhu J, Wei C, ZhuSalzman K, Jin D, Wang J, Liu T, Yu M, Tang C, Wang Z, Dai F, Chen J, Liu Y, Lin T, Zhang S, Yang H, Paterson AH, Xia Q, Ji D, Xiang Z (2013) Draft genome sequence of the mulberry tree Morus notabilis. Nature Communication 4.

Huang Z, Jin SH, Guo HD, Zhong XJ, He J, Li X, Jiang MY, Yu XF, Long H, Ma MD, Chen QB (2016) Genome-wide identification and characterization of TIFY family genes in Moso Bamboo (Phyllostachys edulis) and expression profiling analysis under dehydration and cold stresses. Peer J 4:e2620.

Je J, Song C, Hwang JE, Chung WS, Lim CO (2014) DREB2C acts as a transcriptional activator of the thermo tolerance-related phytocystatin 4 (AtCYS4) gene. Transgenic Research 23:109-123.

Kakkar R, Nagar P, Ahuja P, Rai V (2000) Polyamines and plant morphogenesis. Biologia Plantarum 43:1-11.

Kasukabe Y, He L, Nada K, Misawa S, Ihara I, Tachibana S (2004) Overexpression of spermidine synthase enhances tolerance to multiple environmental stresses and up-regulates the expression of various stress-regulated genes in transgenic Arabidopsis thaliana. Plant and Cell Physiology 45:712-722.

Kunert KJ, van Wyk SG, Cullis CA, Vorster BJ, Foyer CH (2015) Potential use of phytocystatins in crop improvement, with a particular focus on legumes. Journal of Exprimental Botany 66:3559-70.

Levitt J (1980) Responses of Plants to Environmental Stress, Volume 1: Chilling, Freezing, and High Temperature Stresses Academic Press.

Ma B, Luo Y, Jia L, Qi X, Zeng Q, Xiang Z, He N (2014) Genome-wide identification and expression analyses of cytochrome P450 genes in mulberry (Morus notabilis). Journal of Integrative Plant Biology 56:887-901.

Ma X, Chen C, Yang M, Dong X, Lv W, Meng Q (2018) Coldregulated protein (SlCOR413IM1) confers chilling stress tolerance in tomato plants. Plant Physiology and Biochemistry 124:29-39.

Martin-Tanguy J (2001) Metabolism and function of polyamines in plants: recent development (new approaches). Plant Growth Regulation 34:135-148.

Neven LG, Haskell DW, Guy CL, Denslow N, Klein PA, Green LG, Silverman A (1992) Association of 70-kilodalton heat-shock cognate proteins with acclimation to cold. Plant Physiology 99:1362-1369.

Puhakainen T, Hess MW, Mäkelä P, Svensson J, Heino P, Palva ET (2004) Overexpression of multiple dehydrin genes enhances tolerance to freezing stress in Arabidopsis. Plant Molecular Biology 54:743-753.

Rajashekar C, Lafta A (1996) Cell-wall changes and cell tension in response to cold acclimation and exogenous abscisic acid in leaves and cell cultures. Plant Physiology 111:605-612.

Rohela G, Pawan S, Ravindra M, Mudasir G, Aftab A, Srinivasulu Y, Sharma S (2016) Somatic hybridization as a potential tool for mulberry improvement: A review. Indian Horticulture Journal 6:46-49

Rohela GK, Jogam P, Shabnam AA, Shukla P, Abbagani S, Ghosh MK (2018a) In vitro regeneration and assessment of genetic fidelity of acclimated plantlets by using ISSR markers in PPR-1 (Morus sp.): An economically important plant. Scientia horticulturae 241:313-321.

Rohela GK, Shabnam AA, Shukla P, Aurade R, Gani M, Yelugu S, Sharma S (2018b) In vitro clonal propagation of PPR-1, a superior temperate mulberry variety. Indian Journal of Biotechnology 17: 619-625

Rorat T, Szabala BM, Grygorowicz WJ, Wojtowicz B, Yin Z, Rey $P$ (2006) Expression of SK3-type dehydrin in transporting organs is associated with cold acclimation in Solanum species. Planta 224:205-221.

Saeed B, Baranwal VK, Khurana P (2016) Comparative transcriptomics and comprehensive marker resource development in mulberry. BMC Genomics 17:98.

Sakai A, Larcher W (1987) Frost survival of plants: responses and adaptation to freezing stress. 1987, Springer-Verlag, Berlin. 
Sakai A, Yoshida S (1968) The role of sugar and related compounds in variations of freezing resistance. Cryobiology $5: 160-174$.

Shabnam AA, Chauhan S, Khan G, Shukla P, Saini P, Ghosh M (2018) Mulberry breeding strategies for North and North West India. International Journal of Advance Research in Science and Engineering 7:2124-2133

Shen W, Nada K, Tachibana S (2000) Involvement of Polyamines in the Chilling Tolerance of Cucumber Cultivars. Plant Physiology 124:431-440.

Shukla P, Gulab KR, Aftab AS, Sharma SP (2016a) Prospect of Cold Tolerant Genes and Its Utilization in Mulberry Improvement. Indian Horticulture Journal 6: 127-129.

Shukla P, Subhashini M, Singh NK, Ahmed I, Trishla S, Kirti PB (2016b) Targeted expression of cystatin restores fertility in cysteine protease induced male sterile tobacco plants. Plant Science : 246:52-61.

Song X, Sun L, Luo H, Ma Q, Zhao Y, Pei D (2016) GenomeWide Identification and Characterization of Long Non-Coding RNAs from Mulberry (Morus notabilis) RNA-seq Data. Genes (Basel) 7(3): 11. DOI: 10.3390/genes7030011.

Steponkus PL (1984) Role of the plasma membrane in freezing injury and cold acclimation. Annual Review of Plant Physiology 35:543-584.

Subburaj S, Zhu D, Li X, Hu Y, Yan Y (2017) Molecular Characterization and Expression Profiling of Brachypodium distachyon L. Cystatin Genes Reveal High Evolutionary Conservation and Functional Divergence in Response to Abiotic Stress. Frontiers in Plant Science 8, 743. DOI: 10.3389/fpls.2017.00743.

Thomashow MF (1999) Plant cold acclimation: freezing tolerance genes and regulatory mechanisms. Annual review of plant biology 50:571-599.

Uemura M, Joseph RA, Steponkus PL (1995) Cold acclimation of Arabidopsis thaliana (effect on plasma membrane lipid composition and freeze-induced lesions). Plant Physiology 109:15-30.

Ukaji N, Kuwabara C, Takezawa D, Arakawa K, Yoshida S, Fujikawa S (1999) Accumulation of small heat-shock protein homologs in the endoplasmic reticulum of cortical parenchyma cells in mulberry in association with seasonal cold acclimation. Plant Physiology 120:481-490.

Wang F, Hou X, Tang J, Wang Z, Wang S, Jiang F, Li Y (2012) A novel cold-inducible gene from Pak-choi (Brassica campestris ssp. chinensis), BcWRKY46, enhances the cold, salt and dehydration stress tolerance in transgenic tobacco. Molecular Biology Reports 39:4553-64.

Wang Q, Ma B, Qi X, Guo Q, Wang X, Zeng Q, He N (2014) Identification and characterization of genes involved in the jasmonate biosynthetic and signaling pathways in mulberry (Morus notabilis). Journal of Integrative Plant Biology 56:663-72.

Wei C, Liu X, Long D, Guo Q, Fang Y, Bian C, Zhang D, Zeng Q, Xiang Z, Zhao A (2014) Molecular cloning and expression analysis of mulberry MAPK gene family. Plant Physiology \& Biochemistry 77:108-16.

Wisniewski M, Webb R, Balsamo R, Close TJ, Yu XM, Griffith M (1999) Purification, immunolocalization, cryoprotective, and antifreeze activity of PCA60: a dehydrin from peach (Prunus persica). Physiologia of Plant 105:600-608.

Ye H, Du H, Tang N, Li X, Xiong L (2009) Identification and expression profiling analysis of TIFY family genes involved in stress and phytohormone responses in rice. Plant Molecular Biology 71:291-305.

Yoshida S (1984) Chemical and biophysical changes in the plasma membrane during cold acclimation of mulberry bark cells (Morus bombycis Koidz. cv Goroji). Plant Physiology 76:257-265.

Zhang X, Liu S, Takano T (2008) Two cysteine proteinase inhibitors from Arabidopsis thaliana, AtCYSa and AtCYSb, increasing the salt, drought, oxidation and cold tolerance. Plant Molecular Biology 68:131-143.

Zhu D, Bai X, Chen C, Chen Q, Cai H, Li Y, Ji W, Zhai H, Lv D, Luo X (2011) GsTIFY10, a novel positive regulator of plant tolerance to bicarbonate stress and a repressor of jasmonate signaling. Plant Molecular Biology 77:285.

Zhu D, Bai X, Zhu Y, Cai H, Li Y, Ji W, Chen C, An L, Zhu Y (2012) Isolation and functional analysis of GsTIFY11b relevant to salt and alkaline stress from Glycine soja. Hereditas 34:230-239.

Zhu D, Li R, Liu X, Sun M, Wu J, Zhang N, Zhu Y (2014) The positive regulatory roles of the TIFY10 proteins in plant responses to alkaline stress. PLoS One 9(11): e111984. 